\section{Technikfolgenabschätzung zum Raumtransportsystem SÄNGER}

\author{
von Herbert Paschen, Büro für Technikfol- \\ gen-Abschätzung beim Deutschen Bun- \\ destag (TAB)
}

Im Mai 1990 beauftragte der Ausschuss für Forschung, Technologie und Technikfolgenabschätzung des 12. Deutschen Bundestages das Büro für Technikfolgen-Abschätzung (TAB) mit einer Untersuchung zum Raumtransportsystem SÄNGER, dem Leitkonzept des Förderprogramms Hyperschalltechnologie. Der Abschlussbericht des TAB hat maßgeblichen Einfluss auf die Beschlussfassung des Deutschen Bundestages zum HST-Förderprogramm wie auch die Entscheidung der Bundesregierung in dieser Sache gehabt. Darüber hinaus hat der erfolgreiche Abschluss des ersten großen TAB-Projektes ganz wesentlich zur Stabilisierung der Beratungsinstanz des TAB beim Deutschen Bundestag beigetragen.

\section{Zur Bedeutung des Projekts für die dau- erhafte Institutionalisierung des TAB}

Nach langjähriger kontroverser Debatte hatte der Deutsche Bundestag im November 1989 mit der Mehrheit der Regierungsfraktionen von CDU/CSU und F.D.P. beschlossen, den Ausschuss für Forschung und Technologie in „Ausschuss für Forschung, Technologie und Technikfolgenabschätzung" umzubenennen, ihm die Lenkungsfunktion für parlamentsbezogene TAProzesse zu übertragen und eine geeignete wissenschaftliche Einrichtung zu beauftragen, eine ausschließlich für den Deutschen Bundestag arbeitende TA-Einheit aufzubauen und - für einen vertraglich festzulegenden Zeitraum - zu betreiben. Es wurde festgelegt, dass nach einer dreijährigen Erprobungsphase über die weitere Vorgehensweise entschieden werden solle (Bundestags-Drucksachen 11/4606 u. 11/5489).

Um die Durchführung des Modellversuches bewarben sich zahlreiche wissenschaftliche Einrichtungen. Im März 1990 wurde entschieden, der Abteilung für Angewandte Systemanalyse (seit 1995: Institut für Technikfolgenabschätzung und Systemanalyse) des Kernforschungszentrums Karlsruhe (seit 1995: Forschungszentrum Karlsruhe) die Aufgabe zu übertragen, ein „Büro für TechnikfolgenAbschätzung beim Deutschen Bundestag“ (TAB) am Sitz des Deutschen Bundestages aufzubauen und für zunächst drei Jahre zu betreiben. Das Arbeitsprogramm umfasste zu Beginn fünf TA-Projekte:

- Raumtransportsystem SÄNGER

- Risiken bei einem verstärkten Wasserstoffeinsatz

- Grundwasserschutz und Wasserversorgung

- Abfallvermeidung und Hausmüllentsorgung

- Biologische Sicherheit bei der Nutzung der Gentechnik.

Am 4. März 1993 beschloss der Deutsche Bundestag einstimmig (!), die im Rahmen des Modellversuchs aufgebaute TA-Kapazität $a b$ 1. September 1993 in eine ständige Einrichtung des Deutschen Bundestages zu überführen (Bundestags-Drucksache 12/4193). Der Betrieb des TAB durch das Forschungszentrum Karlsruhe wurde auf weitere fünf Jahre (bis 1998) vertraglich sichergestellt. In der Plenardebatte wurden die Beschlussempfehlung und der Bericht des Ausschusses für Forschung, Technologie und Technikfolgenabschätzung zur Institutionalisierungsfrage und die Beschlussempfehlung und der Bericht zum Raumtransportsystem SÄNGER zusammen behandelt. Die Debatte machte deutlich, dass der positive - von allen Fraktionen getragene - Institutionalisierungsbeschluss ganz wesentlich davon beeinflusst wurde, dass die fachliche Qualität und die politische Relevanz der SÄNGER-Studie die Parlamentarier vom Nutzen der neuen Einrichtung für ihre Arbeit überzeugten. Für die CDU/CSU verwies der Abgeordnete Dr. Martin Mayer ausdrücklich auf diesen Zusammenhang (Deutscher Bundestag, 12. Wahlperiode, Sitzungsprotokoll 143. Sitzung, S. 12338-12339). Die SPD-Abgeordnete Edelgard Bulmahn stellte fest: „Bereits mit dem ersten vorgelegten Endbericht, der in dieser Debatte gleichfalls zur Diskussion steht, der TA-Studie zum Raumtransportsystem SÄNGER, landete das TAB, wie ,Die ZEIT' zu Recht feststellte, ,einen Treffer'. Der Bericht stellt in überzeugender Weise den Wert derartiger Studien für den politischen Entscheidungsprozess unter Beweis [...] Die vorliegende Studie (hat) zu einer deutlichen Stärkung der technologischen Kompetenz dieses Hauses beigetragen“ (a. a. O., S. 12335-12337). Der Abgeord 
nete Jürgen Timm erklärte für die F.D.P., dass die SÄNGER-Studie des TAB bei der im Ausschuss für Forschung, Technologie und Technikfolgenabschätzung zu treffenden Entscheidung zur Raumfahrttechnik sehr hilfreich gewesen sei (a. a. O., S. 12343).

\section{Zum Inhalt der SÄNGER-Studie}

Gegenstand des Projekts war die geplante Fortsetzung des deutschen Hyperschalltechnologie(HST)-Förderprogramms und dessen Ausrichtung auf das Leitkonzept SÄNGER. Bei dem Konzept SÄNGER handelt es sich um ein zweistufiges, horizontal startendes und landendes, wieder verwendbares Raumtransportsystem. Die erste Phase des seit 1988 laufenden HST-Förderprogramms sollte nach ursprünglicher Planung 1992 abgeschlossen werden. Da der für den Übergang in die nächste Phase erforderliche Technologiestand bis Ende 1992 aber offenbar nicht erreichbar war, beabsichtigte das BMFT eine Verlängerung der Phase I um voraussichtlich drei Jahre.

Der Abschlussbericht des TAB zur „TA SÄNGER" wurde im Juni 1992 vorgelegt und im Februar 1993 mit einer Beschlussempfehlung des Ausschusses als Bundestagsdrucksache veröffentlicht (Bundestags-Drucksache 12/4277)*. Der Bericht enthält ausführliche Technik-, Bedarfs-, Ziel- und Folgenanalysen, die die Grundlage für die Herausarbeitung und Diskussion politischer Handlungsoptionen bildeten.

\section{Technikanalyse}

Analysiert werden zunächst die zum Untersuchungszeitpunkt verfügbaren bzw. in Entwicklung befindlichen Raumtransportsysteme - mit Ausnahme des Space Shuttle eine breite Palette von „Verlustträgern“ (d. h. nicht wieder verwendbaren Systemen) - und ihre Hauptdefizite, wie z. B. ihr nur geringes Potenzial zur Reduktion der Transportkosten. Aus dieser Defizitanalyse werden Anforderungen an zukünftige Raumtransportsysteme abgeleitet: Wiederverwendbarkeit, Horizontalstart und -landung, luftatmende Antriebe usw. Die wichtigsten zu der Zeit diskutierten Konzepte für Raumtransportsysteme der „,nächsten Generation“ - darunter das Konzept SÄNGER - werden dargestellt. Konzeption, Ablauf und Organisation des deutschen Hyperschalltechnologie-Programms und die mit diesem Programm bzw. einem zukünftigen Raumtransportsystem vom Typ SÄNGER verfolgten Ziele werden beschrieben. Bei diesen Zielen handelt es sich überwiegend um „Optimierungsziele" - Raumtransporte sollten in Zukunft z. B. wirtschaftlicher, sicherer, umweltfreundlicher werden -, die den Bedarf nach Raumtransporten und Raumtransportsystemen voraussetzten.

\section{Bedarfsanalyse}

Die kritische Frage des Bedarfs nach neuartigen Raumtransportsystemen wird ausführlich untersucht. Nach einer Darstellung der kontroversen Diskussion um den wissenschaftlich-technischen und den industrie-, wirtschafts-, außen-, sicherheits- und umweltpolitischen Nutzen der Raumfahrt und der in dieser Diskussion vertretenen Positionen werden zwei raumfahrtpolitische Nutzungsszenarien beschrieben und diskutiert, insbesondere auch im Hinblick auf darin enthaltene mögliche zukünftige ,Marktsegmente" für ein Raumtransportsystem vom Typ SÄNGER. Für ein „konservatives“ Szenario wurde die Gesamtzahl möglicher Starts für ein europäisches Raumtransportsystem vom Typ SÄNGER auf ca. 8 bis 15 pro Jahr geschätzt, davon möglicherweise zwei bis drei bemannte Starts. Diese Zahlen wären mit Sicherheit nicht ausreichend, um auch nur eine Minimalflotte von zwei wieder verwendbaren Transportern vom Typ SÄNGER wirtschaftlich zu betreiben.

Schon die vom Umfang her geringste zusätzliche Aktivität des ,progressiven“ Szenarios, der Aufbau und Betrieb einer permanenten, aber nur gelegentlich bemannten Mondstation, z. B. in Verbindung mit einem Mondobservatorium, würde zu einer Verdopplung des mittleren Transportbedarfs des konservativen Szenarios führen. Der zusätzliche Transportbedarf würde dann für Marsmissionen mit Rückflugmöglichkeit um eine Größenordnung und für Solarenergiesatelliten um drei Größenordnungen ansteigen. Das „progressive“ Szenario mit seinem großen Wachstumspotenzial erschien jedoch unter anderem nur dann wirklich realisierbar, wenn die Transportkosten entscheidend gesenkt werden könnten.

Die Analyse der möglichen zukünftigen „Marktsegmente“, die sich im „konservativen“ und im ,progressiven“ Raumfahrtszenario für 
SÄNGER oder ein anderes fortschrittliches Raumtransportsystem ergeben könnten, zeigte, dass das Konzept SÄNGER auf jeden Fall in scharfer Konkurrenz mit anderen Konzepten für das Raumtransportsystem der ,nächsten Generation" stehen würde und dass Chancen für SÄNGER eigentlich nur im Rahmen eines „progressiven“ Szenarios bestehen würden.

\section{Ziel- und Folgenanalysen}

Im Mittelpunkt stand hier das Ziel der Startkostenreduzierung. Vom Einsatz wieder verwendbarer Raumtransportsysteme wie SÄNGER anstelle von Verlustsystemen versprach man sich eine deutliche Senkung der Startkosten vor allem für kleinere und mittlere Nutzlasten. Grundsätzlich war davon auszugehen, dass die erhoffte Reduzierung der Raumtransportkosten nur erreicht werden kann, wenn es gelänge, die im Vergleich zu Verlustsystemen höheren Entwicklungs- und Baukosten wieder verwendbarer Systeme über relativ hohe jährliche Startzahlen und eine hohe Lebensdauer abzuschreiben und so geringere spezifische Startkosten als bei Verlustsystemen zu erreichen.

Im Rahmen der TA-Studie durchgeführte Modellrechnungen bestätigten dies. Der Breakeven-Punkt bzw. Kostenschnitt mit konventionellen Systemen wie ARIANE 5 hätte sich im Fall von SÄNGER für reine Frachttransporte erst bei relativ hohen Transportleistungen von $150 \mathrm{Mg}$ pro Jahr ergeben, was ca. 20 Starts einer SÄNGER-Flotte pro Jahr entsprechen würde. In einem „konservativen“ Szenario ergäbe sich für ein europäisches Transportsystem vom Typ SÄNGER jedoch, wie schon erläutert, nur ein jährlicher Bedarf von 8 bis 15 Flügen, einschließlich möglicherweise zwei bis drei bemannten Flügen.

Ein weiteres Hauptziel der Entwicklung neuartiger Raumtransportsysteme wie SÄNGER war die Erhöhung der Sicherheit und Zuverlässigkeit des Raumtransports - auf ein Niveau, das dem in der Luftfahrt erreichten möglichst nahe kommt. Die TAB-Studie kommt hier unter anderem zu dem Ergebnis, dass Zweistufer - wie SÄNGER - wegen des unkritischeren Leermassenanteils der Unterstufe Vorteile gegenüber einstufigen Systemen haben. Es wird aber bezweifelt, ob mit Syste- men wie SÄNGER ein Sicherheitsniveau erreicht werden kann, das dem im Bereich der Luftfahrt erreichten nahe kommt.

Weitere Ziele, die in der SÄNGER-Studie überprüft wurden, sind die Verbesserung der Umweltverträglichkeit des Raumtransports, die Möglichkeit der Durchführung von Raumtransporten von europäischen Flugplätzen aus und die Nutzung der Spin-off-Potenziale eines möglichen SÄNGER-Projekts bzw. des Hyperschalltechnologie-Programms.

\section{Entscheidungsbedarf und Handlungsoptionen}

Die Policy-Analyse des TAB führte zum einen zu der Empfehlung an den Deutschen Bundestag, eine politische Grundsatzentscheidung zum Umfang des zukünftigen Engagements Deutschlands bei der Weltraumnutzung vor Eintritt in die nächste Phase des HSTProgramms zu treffen, mit der Begründung, die Weiterentwicklung eines operationellen HSTRaumtransportsystems sei nur in einem ,progressiven" Weltraumszenario sinnvoll.

Zum anderen wurden drei Optionen für die Fortführung des HST-Programms entwikkelt und analysiert:

Eine erste Option für die Zeit nach Ablauf von Phase I des Programms wäre ein ,Moratorium“ für die Projektförderung des BMFT im Bereich der Hyperschalltechnologie gewesen. Diese Option eines "Moratoriums“ hätte zur Folge gehabt, dass die Kontinuität der Technologiearbeiten unterbrochen würde. Andererseits würden Mittel eingespart, falls es später zu einer raumfahrtpolitischen Grundsatzentscheidung gegen einen Einstieg in eine ,progressive“ Weltraumnutzung kommen würde.

Die zweite Option lief darauf hinaus, die Phase I des Hyperschalltechnologie-Programms auf der Basis des Leitkonzepts SÄNGER nach den bestehenden Planungen des BMFT bis 1995 fortzusetzen, aber unter Berücksichtigung bestimmter Ergänzungen und Modifikationen. Ziel dieser Option war die Klärung noch offener technologischer Fragen, die einem Übergang in die Phase II des am Leitkonzept SÄNGER orientierten Programms im Wege standen.

Die dritte Option umfasste im Wesentlichen folgende Punkte: Ausweitung des Technologie-Programms auf kritische Technologien und Schlüsseltechnologien für Raumtransport 
systeme unterschiedlicher Konzeption; Erstellung eines umfassenden Vergleichs der verschiedenen Konzepte für ein zukünftiges Raumtransportsystem; Verbreiterung der internationalen Kooperation; Reduzierung der Arbeiten, die sich spezifisch am Leitkonzept SÄNGER orientierten. Das Technologie-Programm sollte so breit angelegt werden, dass die deutsche Raumfahrtindustrie auch im Falle der (auf europäischer oder internationaler Ebene getroffenen) Wahl eines anderen Konzepts als SÄNGER eine gewichtige technologische Rolle übernehmen könnte.

\section{Zur politischen Relevanz der SÄNGER- Studie}

Die TAB-Studie wurde in der Stellungnahme des zuständigen Ausschusses für Forschung, Technologie und Technikfolgenabschätzung als „wichtige Informationsbasis für die anstehenden Entscheidungen zur Fortsetzung des HSTProgramms und zur zukünftigen Weltraumpolitik der Bundesrepublik Deutschland" bezeichnet (Bundestags-Drucksache 12/4277, S. 5). Im Januar 1993 verabschiedete der Ausschuss einstimmig eine Beschlussempfehlung an den Deutschen Bundestag, in der der Bundesminister für Forschung und Technologie aufgefordert wurde, a) das HST-Programm in der Phase I entsprechend der dritten Option des TAB- Berichtes umzugestalten; b) in Abstimmung mit den europäischen Partnern eine politische Grundsatzentscheidung über den Umfang der zukünftigen europäischen Weltraumnutzung und deren Finanzierung aus öffentlichen Mitteln herbeizuführen. Diese Beschlussempfehlung wurde am 4. März 1993 vom Deutschen Bundestag mit großer Mehrheit angenommen.

Im Dezember 1996 richteten die TABerichterstatter des Forschungsausschusses an den Bundesminister für Bildung, Wissenschaft, Forschung und Technologie eine Anfrage unter anderem folgenden Inhalts: „Welche Maßnahmen wurden von Seiten der Bundesregierung und der Industrie zur Reorientierung des HSTProgramms/Leitkonzept SÄNGER eingeleitet und inwieweit hat die Bundesregierung dabei der TAB-Studie ,Technikfolgenabschätzung zum Raumtransportsystem SÄNGER' [...] bzw. dem Beschluss des Deutschen Bundestages [...] Rechnung getragen?“ Die Bundesregierung
(BMBF) antwortete wie folgt: „Entsprechend den Beschlüssen des Deutschen Bundestages und den Ergebnissen der TAB-Studie „Technikfolgenabschätzung zum Raumtransportsystem SÄNGER “wurden 1993 die Forschungsarbeiten vom Bundesministerium für Bildung, Wissenschaft, Forschung und Technologie (BMBF), der Industrie und der wissenschaftlichen Einrichtungen neu ausgerichtet. Dabei wurde im Zeitraum von 1993 bis 1995 das Technologieprogramm auf wichtige, kritische Entscheidungskomponenten und Schlüsseltechnologien für ein HST-Transportsystem hin orientiert. Die spezifischen Arbeiten zum SÄNGER-Konzept wurden reduziert. [...] Insbesondere über FESTIP (Future European Space Transportation Investigation Programme) [...] wurden die Voraussetzungen zum Beginn einer internationalen Kooperation für das HST- und andere wieder verwendbare Raumtransportsysteme - geschaffen“"(Bundestags-Drucksache 13/6628, S. 45 f.).

Die Untersuchung zum Raumtransportsystem SÄNGER ist ein Beispiel für ein TABProjekt mit hoher Relevanz für parlamentarische Beratungs- und Entscheidungsprozesse. Das Parlament hat eine der vom TAB entwickelten Handlungsoptionen ausdrücklich übernommen. Dies tat auch die Bundesregierung. Zweifellos war das Projekt unter diesem Gesichtspunkt eine der erfolgreichsten Arbeiten des TAB. Ein Erfolg für das TAB war die SÄNGER-Studie im Übrigen auch wegen der breiten - und durchweg positiven - Resonanz in den Medien auf das erste Projekt dieser neuen Einrichtung.

\section{Zur Kooperation zwischen ITAS bzw. AFAS und TAB am Beispiel des SÄNGER-Projekts}

Als die Arbeiten am SÄNGER-Projekt begonnen wurden, war der personelle Aufbau des TAB am Standort Bonn noch nicht abgeschlossen. Da der Projektbericht wegen der Dringlichkeit der politischen Entscheidung über die Fortführung des HST-Programms bis spätestens Mitte 1992 dem Ausschuss vorzulegen war, wurde die Bearbeitung des Projekts - von der Entwicklung des Untersuchungskonzepts über die Vergabe und Betreuung von Gutachten bis zur Abfassung des Endberichts - erfahrenen Mitarbeitern der Abteilung für Angewandte Systemanalyse des Forschungszentrums Karlsruhe übertragen. 
Dass die Abteilung für Angewandte Systemanalyse in der Lage und bereit war, einen solchen Beitrag ,aus dem Stand“ zu erbringen, macht deutlich, wie richtig die politische Entscheidung gewesen war, mit dem Aufbau und Betrieb des TAB eine Einrichtung mit langjähriger Erfahrung in der wissenschaftlichen Politikberatung und einem Stamm von Mitarbeiterinnen und Mitarbeitern aus einem breiten Spektrum ingenieur-, natur-, wirtschafts- und sozialwissenschaftlicher Disziplinen zu beauftragen. Auch das „Multimedia“-Projekt wurde ausschließlich in AFAS bearbeitet. In vielen weiteren Projekten hat sich die Unterstützung des TAB durch Mitarbeiterinnen und Mitarbeiter von AFAS/ITAS als erforderlich erwiesen, wenn auch meist in geringerem Umfang als beim SÄNGER-Projekt. Beispiele sind die Untersuchungen $\mathrm{zu}$ den Themen Integrierte Umwelttechnik (siehe hierzu den Beitrag von Rolf Meyer in diesem Schwerpunkt), Entlastung des Verkehrsnetzes und E-Commerce.

Der damalige stellvertretende Leiter der AFAS, Reinhard Coenen, übernahm mit der Bearbeitung der Kosten- und Wirtschaftlichkeitsanalysen einen besonders schwierigen und kritischen, für die zu treffenden politischen Entscheidungen zentralen Teil des SÄNGERProjekts. Er war darüber hinaus maßgeblich beteiligt an der heiklen Aufgabe der Formulierung und (internen) Bewertung politischer Handlungsoptionen in der überaus hektischen Endphase des Projekts. Dass es trotz aller Hektik zu einem erfolgreichen - und pünktlichen Abschluss des Projekts gekommen ist, ist dem durch Gelassenheit und gesunden Menschenverstand geprägten Einsatz von Reinhard Coenen in hohem Maße zu verdanken.

* Beschlussempfehlung und Bericht des Ausschusses für Forschung, Technologie und Technikfolgenabschätzung zur Technikfolgenabschätzung (TA); hier: Raumtransportsystem SÄNGER. Bundestagsdrucksache 12/4277 vom 4.2.1993

\section{Kontakt}

Prof. Dr. Herbert Paschen

Büro für Technikfolgen-Abschätzung beim Deutschen Bundestag (TAB)

Neue Schönhauser Straße 10, 10178 Berlin

E-Mail: buero@tab.fzk.de

Internet: http://www.tab.fzk.de

।)

\section{Politikberatung bei mehrfacher „Unschärfe“" TAB-Projekt „Integrierte Umwelt- technik“}

\author{
von Dr. Rolf Meyer, TAB/Europäisches \\ Parlament*
}

1993 beauftragte der Deutsche Bundestag das Büro für Technikfolgen-Abschätzung beim Deutschen Bundestag (TAB), die "Bedeutung der Umwelttechnik für die wirtschaftliche Entwicklung der Bundesrepublik Deutschland" zu untersuchen. Intention des Projektes war die Suche nach positiven Perspektiven für den Umweltschutz, d. $h$. es sollten Optionen entwickelt werden, wie durch technologie- und umweltpolitische Maßnahmen die Verbesserung der Umweltqualität positiv beeinflusst werden kann und mögliche negative Wirkungen für den Standort Deutschland vermieden werden können. Der Abschlussbericht des Projektes, der im Jahr 1996 vorgelegt wurde, zeigt Wege auf, wie eine Trendwende vom bisher überwiegenden Einsatz nachsorgender und additiver Umwelttechnik hin zu einer verstärkten Nutzung integrierter Umwelttechniken eingeleitet und gefördert werden könnte unter weitgehender Vermeidung negativer Wirkungen für den Standort Deutschland.

\section{Ausgangslage}

Die umweltpolitische Situation Anfang der 90er Jahre war gekennzeichnet durch erste deutliche Verbesserungen, beispielsweise in den Bereichen Luft- und Wasserreinhaltung und Abfallbeseitigung. Diese Fortschritte beruhten in der Regel auf ordnungsrechtlichen Regelungen, die zum Einsatz additiver Umwelttechniken führten. Gleichzeitig waren durch verschärfte internationale Konkurrenz und in Folge der deutschen Wiedervereinigung ökonomische und sozialpolitische Fragestellungen deutlich in den Vordergrund gerückt. Damit wurde es zunehmend schwieriger, weitere Verbesserungen im Umweltschutz politisch durchzusetzen.

Vor diesem Hintergrund beauftragte der Deutsche Bundestag 1993 das TAB, eine Technikfolgen-Abschätzung zu dem Thema „Die 